\title{
Melhorias no projeto de interface como resultado de avaliações formativas de IHC: o caso de um Sistema Jurídico
}

Interface design improvements as a result of $\mathrm{HCl}$ formative

assessment: the case of a Law Management System

\author{
Rafael Dias Santos \\ Universidade Federal do Rio Grande do Norte \\ rafaeldias.a@gmail.com \\ Bruno Santana da Silva \\ Universidade Federal do Rio Grande do Norte \\ bruno@imd.ufrn.br $\mathbf{x}$
}

\section{PROJËTICA}

\section{COMO CITAR ESTE ARTIGO:}

SANTOS, Rafael Dias; SILVA, Bruno Santana da. Melhorias no projeto de interface como resultado de avaliações formativas de IHC: o caso de um Sistema Jurídico. Projética, Londrina, v. 11, n. 2, p. 80-103, 2020.

DOI: 10.5433/2236-2207.2020v11n2p80

Submissão: 15-07-2019

Aceite: $27-10-2019$ 
RESUMO: Avaliação é uma importante atividade durante a concepção da solução. Apesar de existirem métodos de avaliação específicos para o design digital, pouco se investigou sobre seus efeitos no processo de design. Este trabalho apresenta um estudo de caso que analisou o uso de resultados de avaliação formativa com avaliação heurística, método de avaliação de comunicabilidade e teste de usabilidade sobre a revisão da solução sendo concebida. Contribuições da avaliação foram evidenciadas e questões sobre a revisão da solução precisam de investigações no futuro.

Palavras-chave: Avaliação de interface com usuário. Melhoria de interface. Usabilidade. Comunicabilidade.

ABSTRACT: The evaluation is an important step while designing new solutions. Although there are specific evaluation methods for digital design, little is investigated about their effects on the design process. This work presents a case study which analyzed the results on the use of formative evaluation with the heuristic evaluation, the communicability evaluation method and the usability test on the review of the designing activity. Contributions of the evaluation have been pointed out and questions about the solution review need further research in the future.

Keywords: User interface evaluation. Interface improvement. Usability. Communicability.

\section{INTRODUÇÃO}

O processo de design é um processo de aprendizado sobre o problema e sobre a solução sendo concebida (SCHÖN, 1983). Independente da metodologia utilizada, a atividade de avaliação é fundamental para apoiar o aprendizado do designer, principalmente sobre a solução projetada (LAWSON, 2006). No âmbito digital, a 
literatura de Interação Humano-Computador (IHC) apresenta uma diversidade de métodos de avaliação (BARBOSA; SILVA, 2010; SHARP; ROGERS; PREECE, 2007). Esses métodos têm sido amplamente utilizados na academia e na indústria e são reconhecidos como boas orientações, um bom apoio, para parte do trabalho do designer.

A avaliação de IHC pode ter vários objetivos. Barbosa e Silva (2010) destacam quatro tipos deles: compreender a apropriação de tecnologia pelos usuários, comparar ideias e alternativas de design, verificar a conformidade com um padrão e identificar problemas na interação e na interface. Na prática do design digital, avaliações de IHC são provavelmente mais realizadas para identificar problemas na interação e na interface de modo a auxiliar o designer encontrar oportunidades de melhoria na sua solução.

Apesar dessa relevância para o processo de design, poucas pesquisas anteriores abordaram o emprego dos métodos de avaliação de IHC (BIM; SALGADO; LEITÃO, 2016; JEFFRIES et al., 1991; KARAT; CAMPBELL; FIEGEL, 1992; SALGADO; BIM; SOUZA, 2006). A maioria delas faz uma análise quantitativa dos problemas encontrados. É importante investigar mais sobre o emprego de métodos de avaliação de IHC como apoio ao processo de design, em particular no que diz respeito à melhoria da solução sendo concebida.

Santos e Silva (2019) apresentaram um estudo de caso (STAKE, 1995; YIN, 2011) que analisou as primeiras experiências de profissionais de uma empresa de tecnologia da informação em avaliações formativas de IHC. Esta empresa startup possuía um sistema web consolidado para gestão de escritórios de advocacia. O estudo ocorreu durante o projeto de interface com usuário de uma versão móvel deste sistema para smartphone. Os colaboradores da empresa, incluindo o autor do projeto de interface, executaram três métodos para identificar problemas no protótipo executável da solução de IHC sendo concebida: avaliação heurística 
(NIELSEN, 1994), método de avaliação de comunicabilidade (PRATES; SOUZA; BARBOSA, 2000) e teste de usabilidade (RUBIN; CHISNELL, 2008). Esse estudo de caso analisou a execução destes métodos considerando tempo, materiais, avaliadores e usuários necessários, resultados encontrados e dificuldades enfrentadas. Santos e Silva (2019) não analisaram como os resultados de avaliação contribuíram para o processo de design.

Este artigo apresenta uma continuação do estudo de caso relatado por (SANTOS; SILVA, 2019), com o objetivo de analisar como os resultados de avaliações de IHC influenciaram melhorias no projeto de interface de um sistema jurídico para smartphone.

\section{MÉTODOS DE AVALIAÇÃO DE IHC}

Os três métodos de avaliação de IHC utilizados neste estudo de caso são brevemente descritos a seguir. Avaliação Heurística $(\mathrm{AH})$ é um método de inspeção que orienta um grupo de avaliadores a navegarem pela interface em busca de potenciais problemas de usabilidade, com base num conjunto de heurísticas (NIELSEN, 1994). Com uma lista de problemas encontrados individualmente, eles se reúnem para revisar os resultados individuais e gerar um relatório coletivo consolidado.

O Método de Avaliação de Comunicabilidade (MAC) é um método de observação de experiências de uso do sistema para identificar problemas de comunicabilidade (PRATES; SOUZA; BARBOSA, 2000). Na preparação, o avaliador define tarefas a serem executadas por potenciais usuários e o perfil dos participantes, faz o recrutamento, prepara o material necessário e executa um teste-piloto. Para coletar dados, o avaliador observa cada participante executando as tarefas solicitadas em ambiente controlado, com gravação do vídeo de interação, e aplica questionário pré e pós-teste. Na interpretação dos vídeos, o avaliador 
marca rupturas de comunicação usando etiquetas com expressões de dúvidas comuns dos usuários. Os resultados são consolidados com a análise de todas as rupturas de comunicação identificadas e a elaboração de um perfil semiótico.

O Teste de Usabilidade (TU) é um método de observação que busca identificar problemas de usabilidade durante a execução de tarefas usando o sistema (RUBIN; CHISNELL, 2008). O TU não se distingue de forma significativa do MAC até a preparação e coleta de dados. As distinções ocorrem nas próximas atividades. Para interpretar os dados e consolidar os resultados, o avaliador realiza medições e sumariza os resultados encontrados. Esta costuma ser apenas uma análise quantitativa, apesar de alguns autores defenderem também uma análise qualitativa no TU.

\section{ESTUDO DE CASO}

Tabela 1 - Quantidade problemas encontrados em cada método

\begin{tabular}{llll}
\hline & total & problemas únicos & problemas sobrepostos \\
\hline $\mathrm{AH}$ & 27 & 18 & 9 \\
\hline $\mathrm{MAC}$ & 20 & 8 & 12 \\
\hline $\mathrm{TU}$ & 5 & 1 & 4 \\
\hline Total & 52 & 27 & 25 \\
\hline
\end{tabular}

Fonte: Santos e Silva (2019).

Santos e Silva (2019) compararam o emprego dos métodos AH, MAC e TU na avaliação formativa da interface do protótipo executável de um sistema jurídico para smartphone. O próprio autor da interface atuou como avaliador na execução destes métodos. No que diz respeito aos resultados da avaliação, Santos e Silva (2019) apresentam o quantitativo de problemas de IHC identificados em cada 
método conforme a Tabela 1. Ao compararem os locais de ocorrência dos problemas encontrados, eles identificaram problemas únicos, identificados por apenas um método, e problemas sobrepostos, identificados por mais de um método no mesmo local. Contudo, eles não se aprofundaram na análise desses resultados, nem em como eles foram utilizados para a melhoria da interface sendo projetada.

Este trabalho tem por objetivo continuar a análise do estudo de caso de (SANTOS; SILVA, 2019), examinando como os resultados da avaliação formativa foram utilizados durante o processo de design na melhoria da solução de IHC sendo concebida. O primeiro passo desta nova etapa do estudo foi estratificar os problemas encontrados por tela e por parte específica dela em que os problemas ocorreram. A Tabela 2 apresenta a estratificação dos problemas encontrados.

Tabela 1 - Quantidade de problemas por local na interface

\begin{tabular}{llll}
\hline tela & parte específica & quantidade & problemas \\
\hline principal & slide (carrossel) & 1 & $\cdot$ AH-1 \\
\hline inscrever-se & calendário & 7 & $\cdot$ AH-11, AH-13, AH-14 \\
& & & MAC-1, MAC-2 e \\
& & & $\cdot$ TU-1 \\
\hline inscrever-se & fluxo de interação & 1 & $\cdot$ AH-2 \\
\hline processos & título da publicação & 2 & $\cdot$ AH-15 e AH-16 \\
\hline processos & detalhes do processo & 2 & $\cdot$ MAC-6 e MAC-7 \\
\hline processos & botão expandir processos & 1 & $\cdot$ AH-3 \\
\hline processos & loading & 1 & $\cdot$ AH-17 \\
\hline processos & botão minimizar tribunais & 1 & $\cdot$ AH-27 \\
\hline processos & calendário & 1 & $\cdot$ MAC-27 \\
\hline processos & lista de processos & 1 & $\cdot \mathrm{MAC}-5$ \\
\hline agenda & calendário & 2 & $\cdot$ AH-4 \\
\hline agenda & busca geral & 2 & $\cdot \mathrm{MAC}-8$ \\
\hline agenda & lista de eventos & 1 & $\cdot \mathrm{AH}-5$ \\
\hline & & $\mathrm{MAC}-10$ \\
\hline
\end{tabular}




\begin{tabular}{|c|c|c|c|}
\hline agenda & botão fechar detalhe evento & 1 & - MAC-9 \\
\hline \multirow[t]{3}{*}{ arquivos } & calendário de arquivos & 3 & $\cdot \mathrm{AH}-6$ \\
\hline & & & - MAC-11 \\
\hline & & & $\cdot$ TU-2 \\
\hline arquivos & botão cancelar download & 1 & $\cdot \mathrm{AH}-7$ \\
\hline arquivos & ícones de arquivos & 1 & - $\mathrm{AH}-19$ \\
\hline \multirow[t]{2}{*}{ contatos } & detalhes do contato & 3 & - $\mathrm{AH}-20$ \\
\hline & & & - MAC-12 e MAC-13 \\
\hline contatos & fonte & 1 & $\cdot \mathrm{AH}-8$ \\
\hline contatos & função do ícone do contato & 1 & • $\mathrm{AH}-21$ \\
\hline contatos & barra lateral de contatos & 1 & • $\mathrm{AH}-25$ \\
\hline \multirow[t]{2}{*}{ geral } & calendário & 2 & • $\mathrm{AH}-10$ \\
\hline & & & - MAC-18 \\
\hline geral & nova tela & 2 & - $\mathrm{AH}-22$ e $\mathrm{AH}-26$ \\
\hline \multirow[t]{2}{*}{ geral } & botões de voltar e avançar & 2 & - $\mathrm{AH}-23$ \\
\hline & & & - MAC-15 \\
\hline geral & busca geral & 2 & - MAC-14 e MAC-16 \\
\hline geral & menu do topo & 1 & - $\mathrm{AH}-9$ \\
\hline geral & ajuda & 1 & - $\mathrm{AH}-12$ \\
\hline geral & botão do Android & 1 & - $\mathrm{AH}-24$ \\
\hline outro & interação & 1 & $\cdot$ TU-3 \\
\hline \multirow[t]{2}{*}{ outro } & domínio & 2 & - MAC-17 \\
\hline & & & • TU-5 \\
\hline outro & equívoco do usuário & 1 & - MAC-19 \\
\hline \multirow[t]{2}{*}{ outro } & erro de programação & 2 & - MAC-20 \\
\hline & & & $\cdot$ TU-4 \\
\hline
\end{tabular}

Fonte: Autores.

Uma tela indica a parte da interface que aborda determinado assunto. Por exemplo, a interface tem uma tela para abordar processos e outra para agenda. Ainda que cada tela se modifique durante a interação usuário-sistema, o assunto principal permanece. Nas situações em que o problema encontrado nas avaliações aconteceu em várias telas ou em toda a interface, a tela foi indicada como "geral" na Tabela 2. Na maioria das vezes, os problemas ocorreram em partes específicas 
da interface relacionadas a widgets, elementos de interação como botões e caixas de texto, por exemplo. Nos poucos casos em que os problemas identificados se referem a outros aspectos envolvidos no uso do sistema diferentes da interface com usuário, a tela foi indicada como "outro" e a parte específica faz referência à interação, ao domínio (falta de conhecimento do usuário sobre os assuntos jurídicos abordados na interface), a equívocos do usuário e a erros de programação (do protótipo utilizado na avaliação), na Tabela 2.

Foram identificadas 32 partes da solução de IHC sendo projetada com algum problema. Apenas um problema foi identificado em 19 partes da solução e mais de um problema foram identificados nas outras 13 partes. AH e MAC encontraram mais de um problema cada em 6 partes da solução avaliada. O TU, não encontrou mais de um problema em nenhuma parte da solução avaliada. Depois de compreender os problemas identificados nas avaliações de IHC realizadas, este estudo analisou como o designer propôs melhorias no protótipo projetado a partir dos problemas identificados nestas avaliações.

\section{REPROJETO DE INTERFACE}

Com base nos resultados dos três métodos de avaliação de IHC, a interface do protótipo projetado foi revisada conforme apresentado a seguir. Cada problema na solução foi indicado como comentário na interface original à esquerda, referenciando o método de avaliação de origem. A correção dos problemas encontrados está indicada à direita, com comentários explicativos. Tanto os comentários na versão original como na versão corrigida foram feitos pelo designer, que também atuou como avaliador da sua própria solução. A Figura 1 indica à esquerda o Problema 1 encontrado na Avaliação Heurística (AH-1) na tela inicial. A respetiva solução proposta foi modificar o comportamento da interface conforme o comentário do designer à direita (S-AH-1, $\mathrm{S}$ de solução). 
Figura 1 - Soluções para problemas de interface na tela inicial

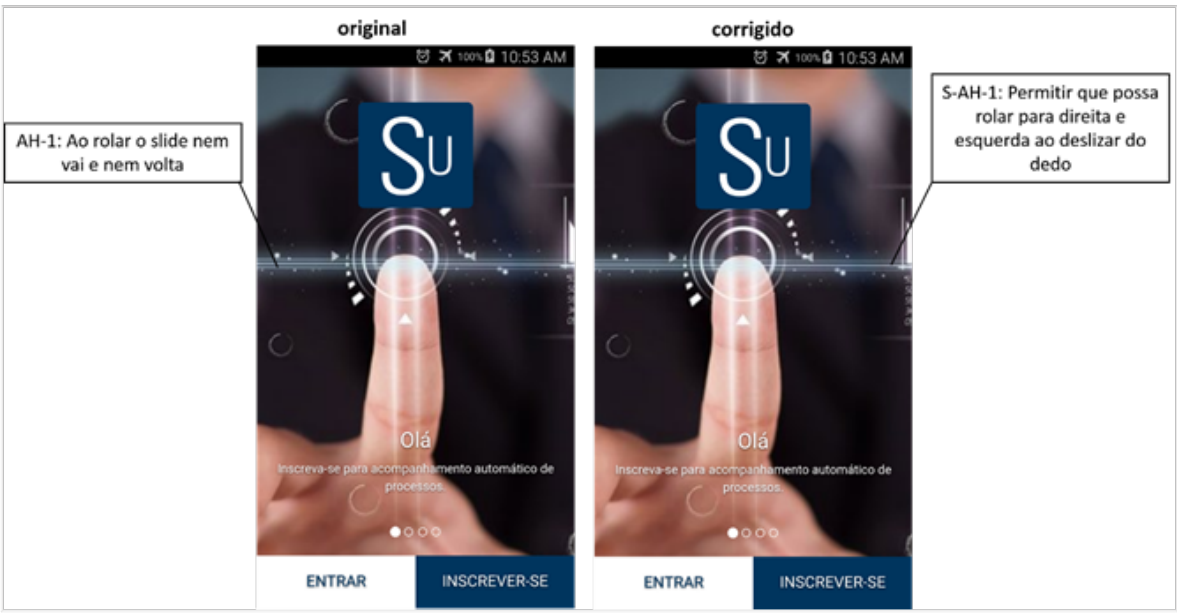

Fonte: Autores.

Figura 2 - Soluções para problemas de interface na tela de inscrever-se

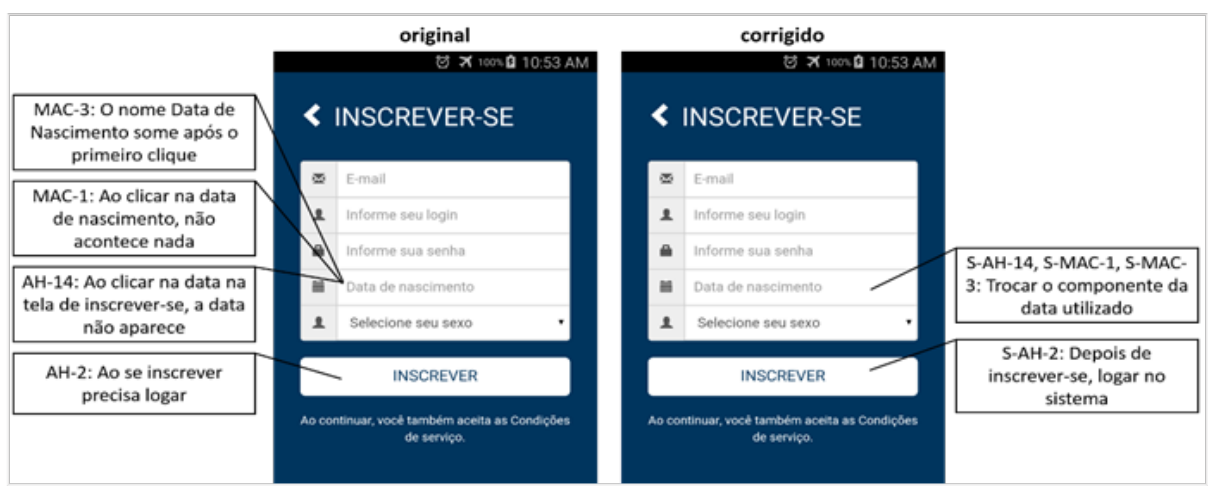

Fonte: Autores.

Todos os métodos permitiram a identificação de problemas na tela onde o usuário pode se cadastrar no sistema (inscrever-se). A Figura 2 aponta 2 problemas identificados pela avaliação heurística ( $\mathrm{AH}-14$ e $\mathrm{AH}-2)$ e mais 2 pelo método de 
avaliação de comunicabilidade (MAC-3 e MAC-1). É preciso alterar o comportamento do widget data de nascimento para abrir um calendário (S-AH-14, S-MAC-1, S-MAC-3) e encaminhar a interação para o login após o cadastro do usuário (S-AH-2). A Figura 3 destaca problemas identificados pela avaliação heurística ( $\mathrm{AH}-11$ e $\mathrm{AH}-13)$, método de avaliação de comunicabilidade (MAC-2) e teste de usabilidade (TU-1). A solução para estes problemas requer uma reformulação completa do widget de calendário, semelhante ao da direita.

Figura 3 - Percentual das áreas de pesquisas dos artigos revisados

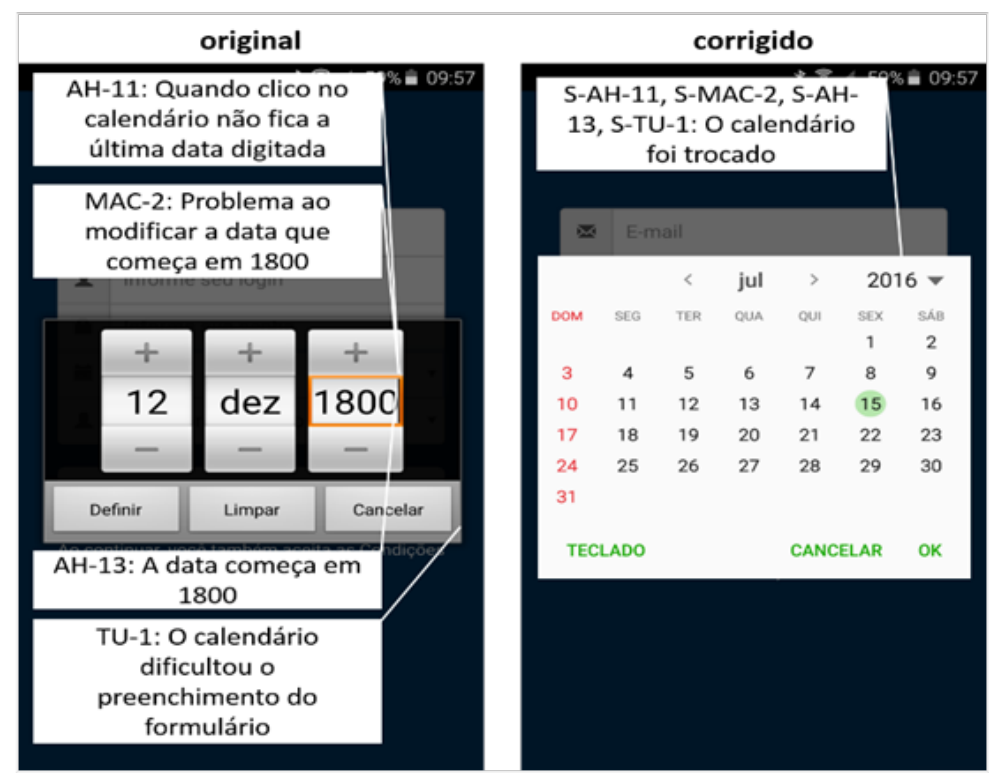

Fonte: Autores.

Apenas a avaliação heurística e o método de avaliação de comunicabilidade orientaram a identificação de problemas relacionados à interface de processos. $A$ Figura 4 destaca 2 problemas identificados pela avaliação heurística (AH-17 e AH-10) e outros 2 pelo método de avaliação de comunicabilidade (MAC-4 e MAC-18). Suas 
soluções envolvem a reformulação do acesso (comportamento ao clicar no "botão" correspondente) e o próprio widget de calendário (S-AH-7, S-AH-10 e S-MAC-4), para permitir visualizações por períodos de tempo (diário, semanal, mensal e anual), bem como preparar um tutorial explicativo (S-MAC-18).

Figura 4 - Soluções para problemas de interface na tela de processos

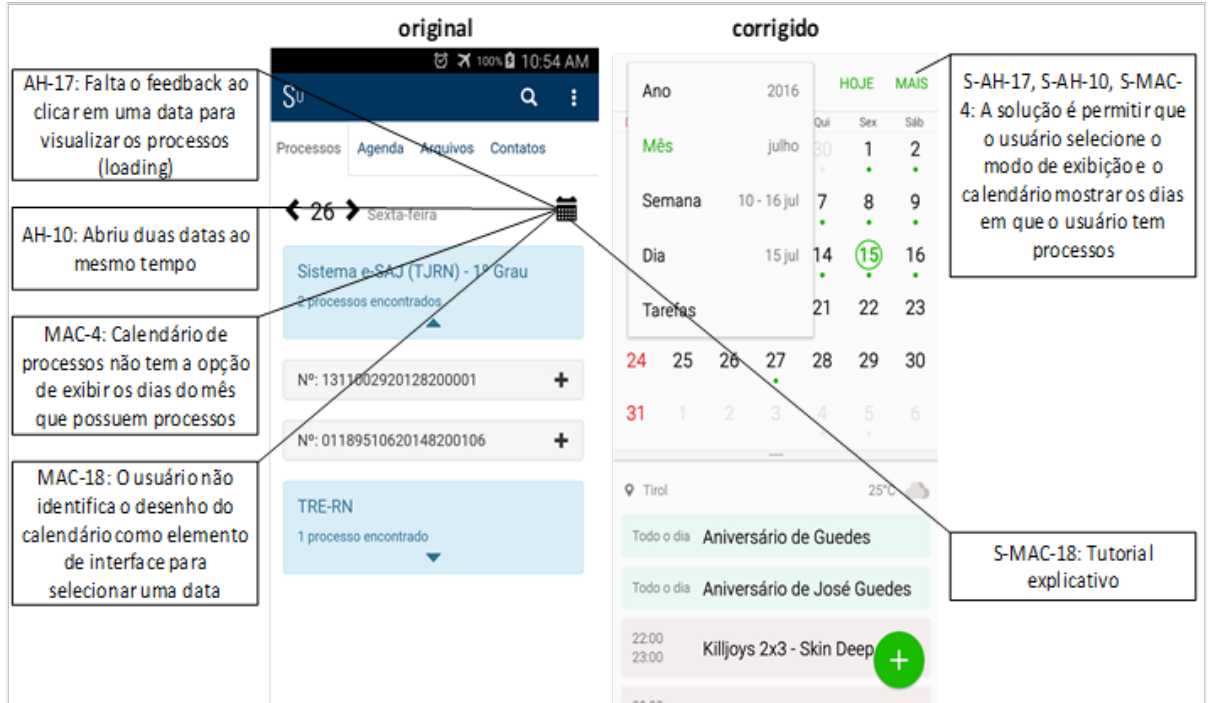

Fonte: Autores.

A Figura 5 apresenta problemas relacionados à visualização de processos, com seus andamentos e publicações. Três problemas foram encontrados pela avaliação heurística ( $\mathrm{AH}-3, \mathrm{AH}-27$ e $\mathrm{AH}-18)$ e um foi encontrado pelo método de avaliação de comunicabilidade (MAC-5). A solução destes problemas envolve oferecer ao usuário a possibilidade de expandir e minimizar todos os processos (S-AH-3, S-AH-27), indicar a quantidade de publicações e andamentos no processo (S-MAC-5) e exibir processos expandidos por padrão (S-AH-18). 
Melhorias no projeto de interface... Jurídico SANTOS, R. D.; SILVA, B. S. da

A avaliação de IHC pode ter vários objetivos. Barbosa e Silva (2010) destacam quatro tipos deles: compreender a apropriação de tecnologia pelos usuários, comparar ideias e alternativas de design, verificar a conformidade com um padrão e identificar problemas na interação e na interface.

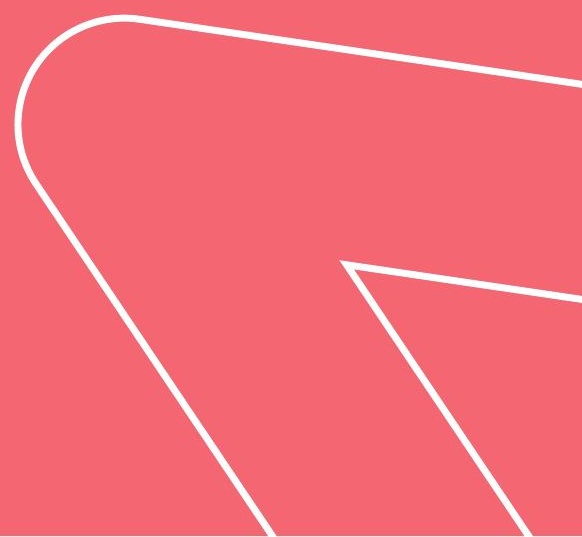


Figura 5 - Soluções para problemas de interface na tela de processos

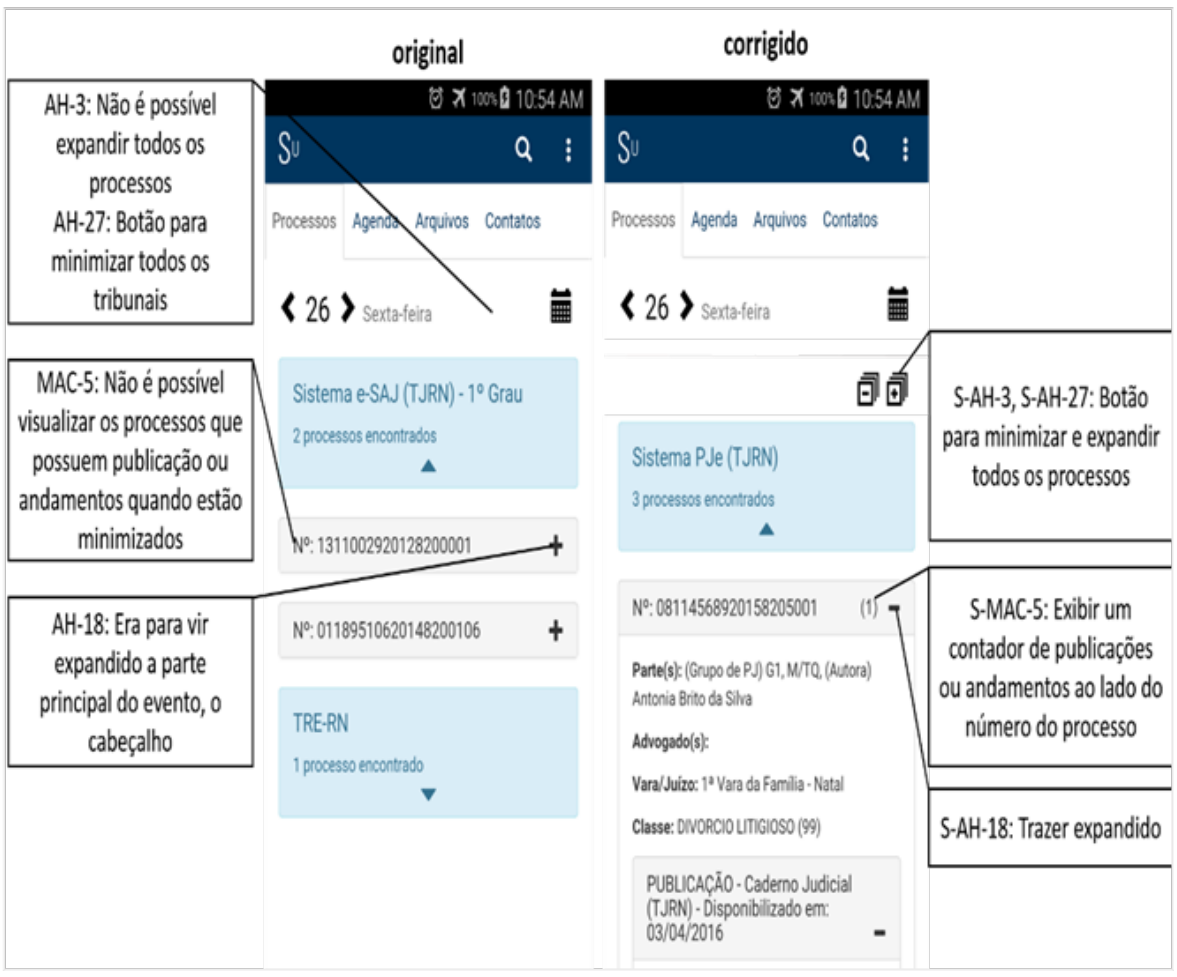

Fonte: Autores.

A Figura 6 apresenta problemas na visualização do processo detalhado. Foram encontrados 3 problemas pela avaliação heurística ( $\mathrm{AH}-9, \mathrm{AH}-15$ e $\mathrm{AH}-16)$ e mais 2 problemas pelo método de avaliação de comunicabilidade (MAC-7 e MAC-6). As soluções desses problemas envolvem: deixar o menu superior sempre visível (S-H-9); relacionar eventos a processo e permitir a navegação entre eles (S-MAC-7); distinguir visualmente publicações e andamentos (S-AH-15); revisar a formatação dos textos nos títulos de publicação e andamento (S-AH-16); e ajustar a rolagem da página automaticamente para manter o foco na parte expandida (S-MAC-6). 
Figura 6 - Soluções para problemas de interface na tela de processos

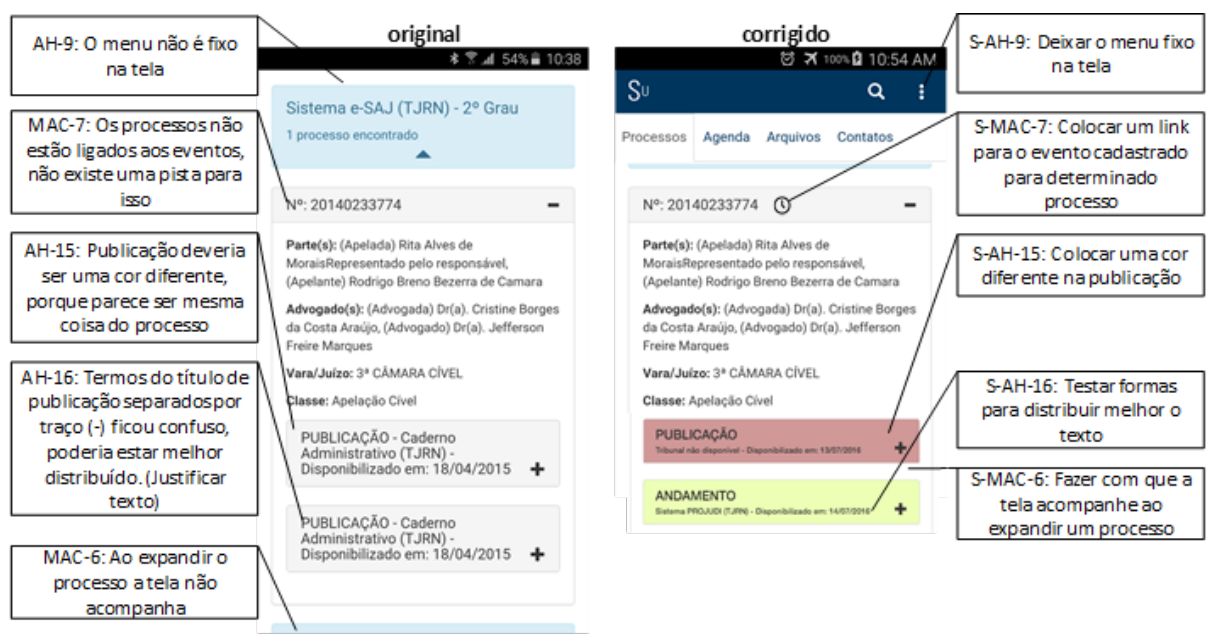

Fonte: Autores.

Figura 7 - Soluções para problemas de interface na tela da agenda
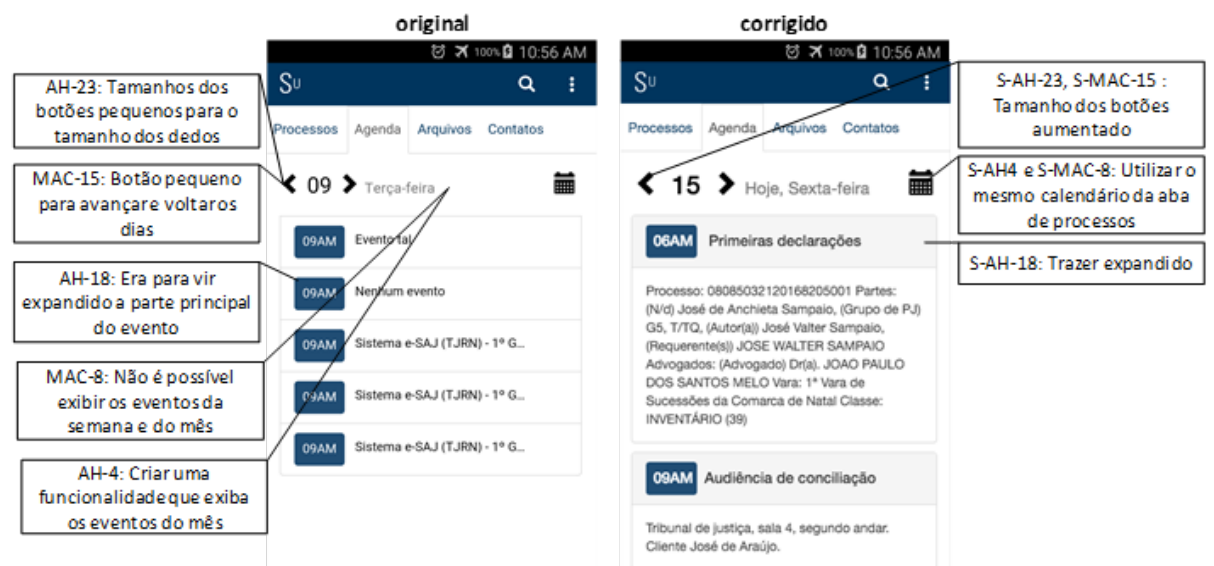

Fonte: Autores. 
$\mathrm{Na}$ interface de agenda, somente a avaliação heurística e o método de avaliação de comunicabilidade orientaram a identificação de problemas. A Figura 7 apresenta 3 problemas encontrados pela avaliação heurística (AH-23, AH-18 e $\mathrm{AH}-4$ ) e outros 2 problemas pelo método de avaliação de comunicabilidade (MAC15 e MAC-8). As soluções envolvem: aumentar o tamanho dos botões (S-AH23 e S-MAC-15); permitir selecionar eventos por outros períodos (diário, semanal, mensal e anuais) semelhante à solução de processos (S-HA-4 e S-MAC-8); e apresentar eventos expandidos por padrão (S-AH-18).

A Figura 8 apresenta 3 problemas identificados pelo método de avaliação de comunicabildiade (MAC-9, MAC-10 e MAC-16). O problema MAC-9 já foi solucionado por S-AH-18, quando definiu que a visualização dos detalhes de um evento seria por expandir e colapsar, e não mais com uma outra tela com voltar. Os outros problemas (MAC-10 e MAC-16) serão resolvidos por um tutorial explicativo para melhorar a metacomunicação (S-MAC-10 e S-MAC-16).

Figura 8 - Soluções para problemas de interface na tela de detalhe de evento

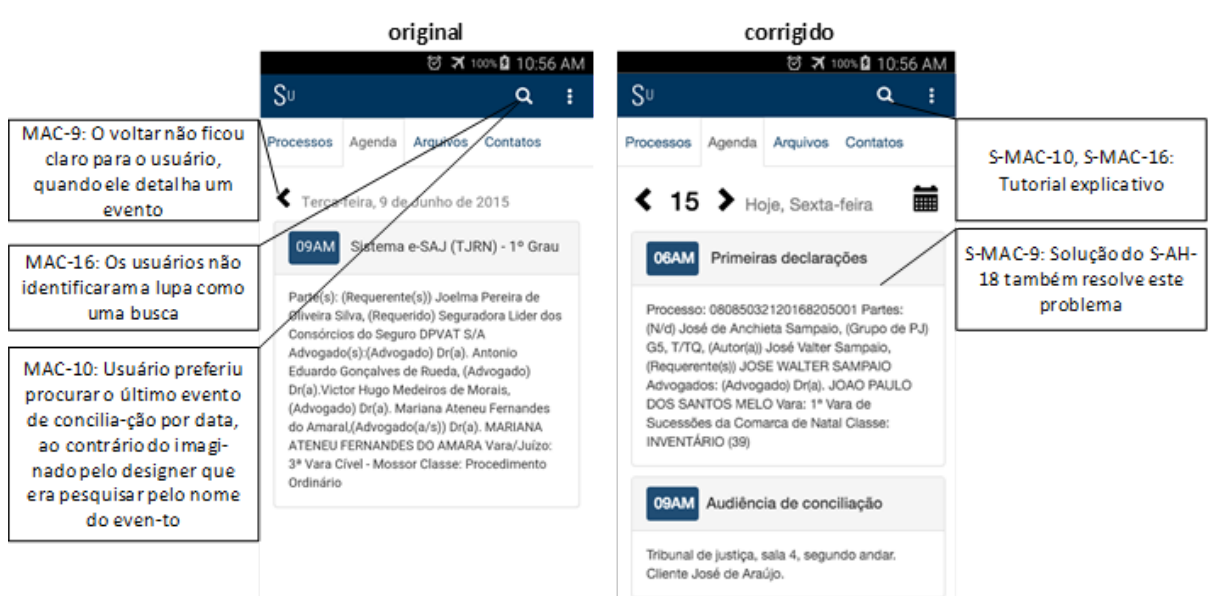

Fonte: Autores. 
A Figura 9 apresenta um problema identificado pela avaliação heurística (AH-5) e outro pelo método de avaliação de comunicabilidade (MAC-14). A solução de ambos envolve indicar a quantidade de eventos encontrados na busca e atualizála corretamente (S-AH-5 e S-MAC-14).

Figura 9 - Soluções para problemas de interface na tela de pesquisa da agenda

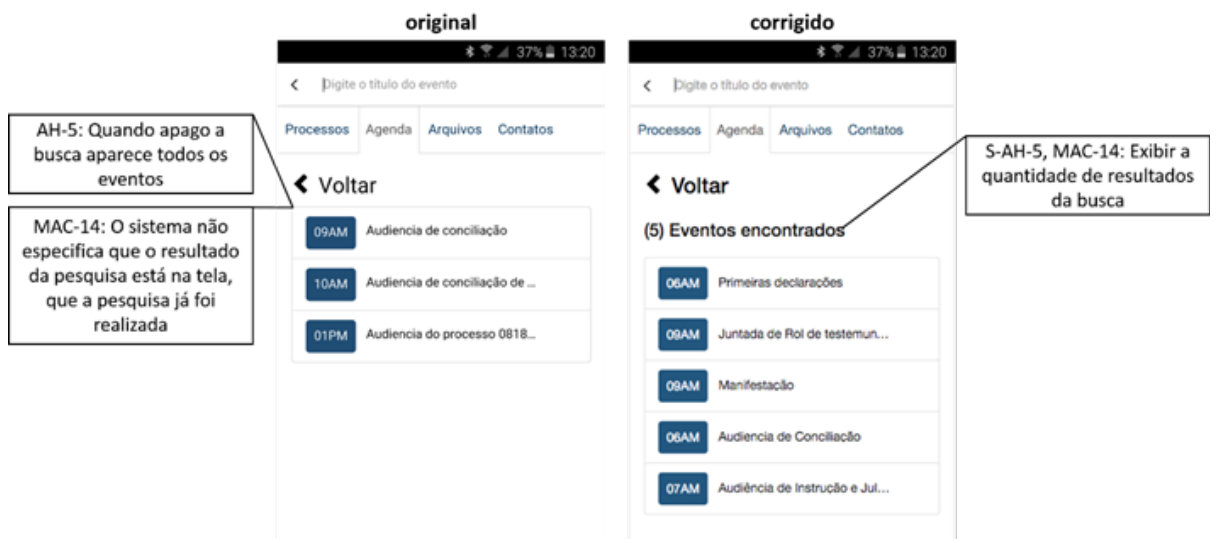

Fonte: Autores.

$\mathrm{Na}$ interface de arquivos, foram encontrados problemas pela avaliação heurística, método de avaliação de comunicabilidade e teste de usabilidade. A Figura 10 apresenta 2 problemas identificados pela avaliação heurística (AH-6 e AH-19), 1 problema pelo método de avaliação de comunicabilidade (MAC-11) e 1 problema pelo teste de usabilidade (TU-2). A solução para os problemas AH-6, MAC-11 e TU-2 é seguir o mesmo padrão de outras partes semelhantes do sistema, permitindo que o usuário filtre os arquivos por datas. Já o problema AH-19 pode ser solucionado com a representação dos tipos de arquivos pelos ícones. A Figura 11 apresenta um problema identificado pela avaliação heurística (AH-7), que foi solucionado com a disponibilização de um botão cancelar o download. 
Figura 10 - Soluções para problemas de interface na tela de arquivos

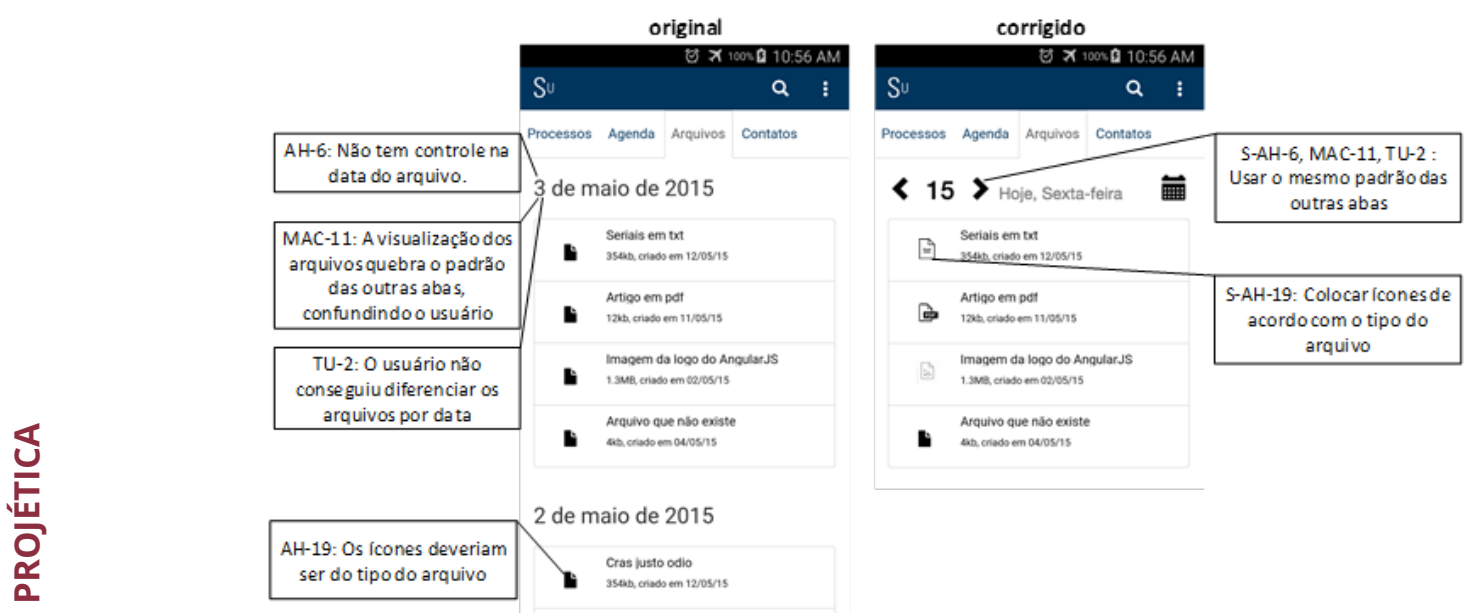

Fonte: Autores.

Figura 11 - Soluções para problemas de interface na tela de download de arquivo
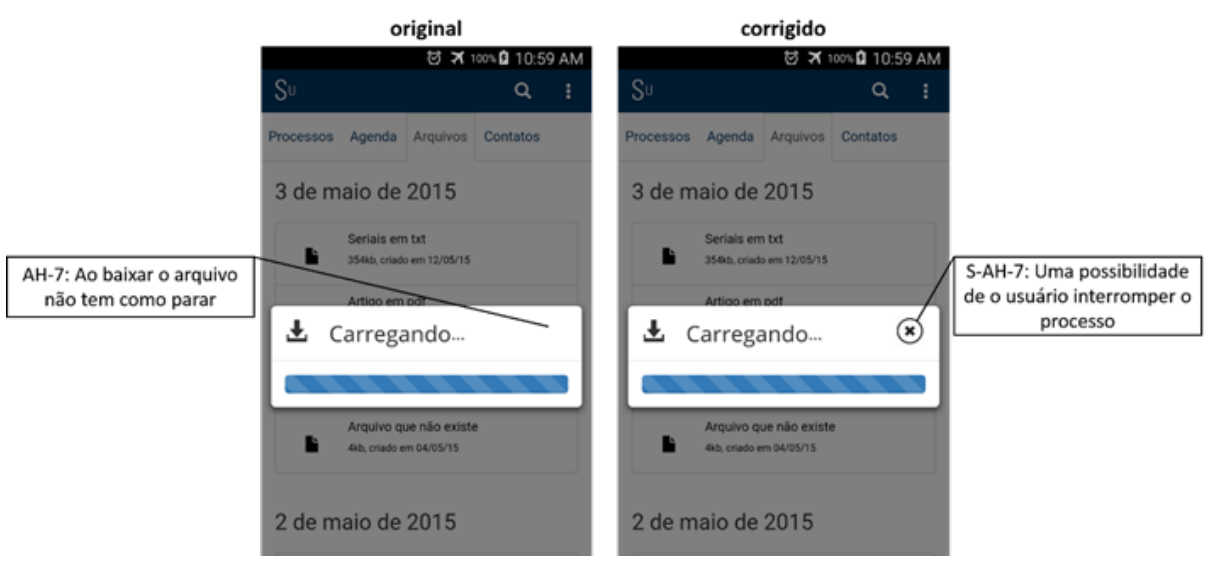

Fonte: Autores.

O teste de usabilidade novamente não auxiliou o avaliador na identificação de problemas relacionados a contatos. A Figura 12 apresenta 3 problemas identificados pela avaliação heurística e 1 problema identificado pelo método de avaliação de 
comunicabilidade. As soluções envolveram: permitir que o usuário defina uma foto para o contato (S-AH-21); ajustar área da interface sensível ao clique sobre o contato (S-MAC-13); ajustar a interface para percorrer mais rápido sobre as letras inicias dos contatos (S-AH-25); e corrigir acentuação dos nomes (S-AH-8).

Figura 12 - Soluções para problemas de interface na tela de contatos

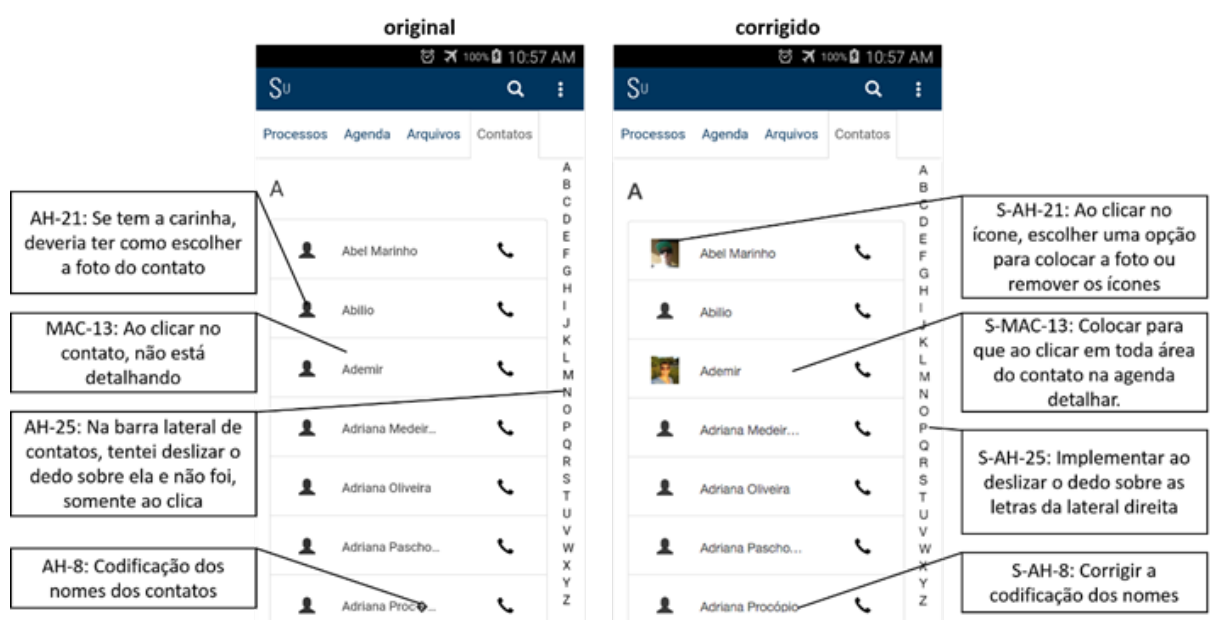

Fonte: Autores.

Figura 13 - Soluções para problemas de interface na tela de detalhes de contato

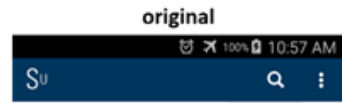

Processos Agenda Arquivos Contatos

< 2 Abel Marinho

AH-20: Nome repetidos ao detalhar um contato

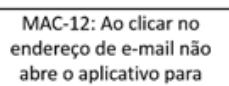

abre $o$ aplicativo para envio do e-mail

Telefones

Yelefone: (051) 8888-8700

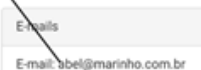

$\zeta$

$\bar{x}$

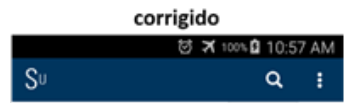

Processos Agenda Arquivos Contatos

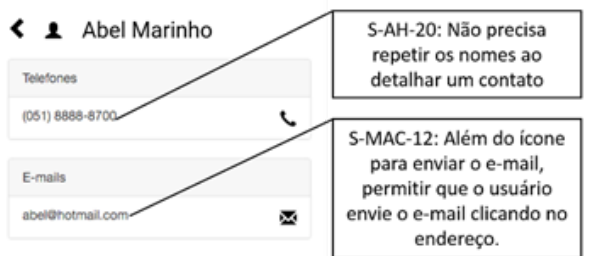

Fonte: Autores. 
A Figura 13 apresenta um problema identificado pela avaliação heurística (AH-20) e outro pelo método de avaliação de comunicabilidade (MAC-12). A solução envolveu remover os termos "telefone" e "e-mail" que estavam repetidos (S-AH-20) e permitir que o usuário clique também no número de telefone e no endereço de e-mail para ativar os respectivos comandos (S-MAC-12).

A Figura 14 apresenta dois problemas identificados pela avaliação heurística. A solução do primeiro requer a definição de perfil do usuário acessado via menu (S-AH-26). O segundo problema requer a criação de ajuda online acessada via menu (S-AH-12).

Figura 14 - Problemas e soluções para a interface em geral

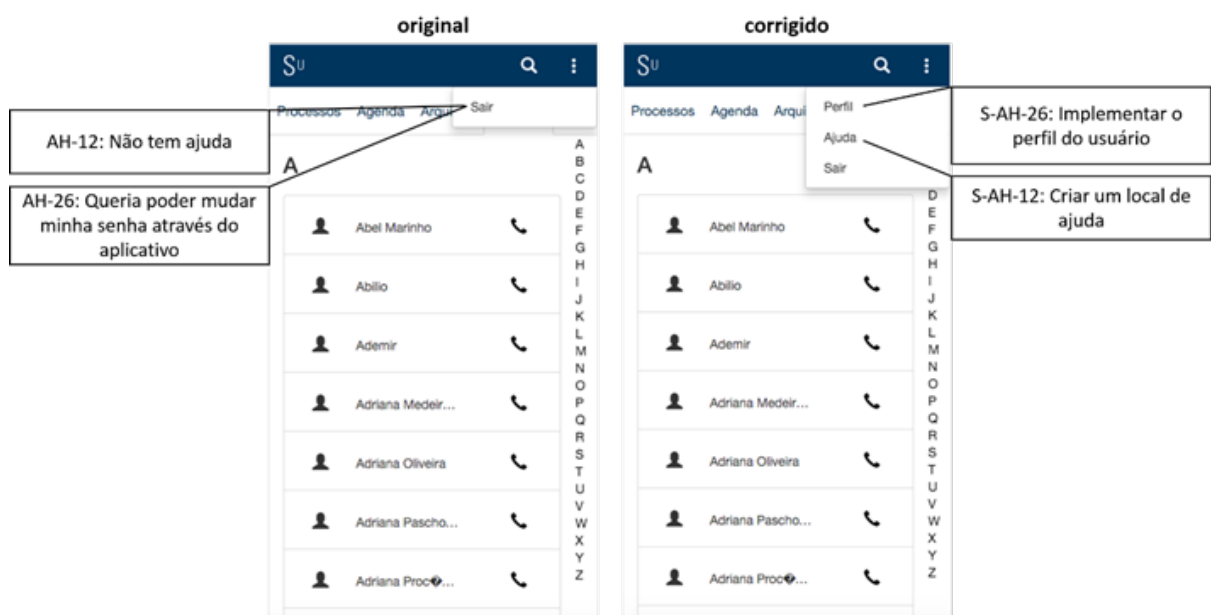

Fonte: Autores.

Nessas correções do projeto foram considerados apenas problemas na interação e interface (46 problemas, $88 \%$ do total) encontrados durante a avaliação formativa do protótipo. Os demais problemas (6 problemas com tela igual a "outro" na Tabela 2, 12\% do total) ainda precisam ser considerados. Os 
resultados da avaliação heurística e do método de avaliação de comunicabilidade contribuíram significativamente para a melhoria da interface, tanto pelos problemas identificados quanto pelas sugestões de melhorias. Já o teste de usabilidade não trouxe contribuições significativas, pois o avaliador teve dificuldade de identificar problemas na interação e interface a partir dos sintomas analisados pelo método.

Um problema identificado na avaliação formativa encaminhou uma modificação na solução de IHC na maior parte das vezes. Em situações particulares, o designer endereçou em conjunto mais de um problema identificado na avaliação quando modificou sua solução de IHC para melhorá-la. Geralmente os problemas foram resolvidos em conjunto quando:

- representam o mesmo problema encontrado por mais de um método de avaliação. Por exemplo, o tamanho pequeno dos botões de navegação pelas datas encontrado pela avaliação heurística ( $\mathrm{AH}-23)$ e pelo método de avaliação de comunicabilidade (MAC-15) na Figura 7;

- os problemas ocorreram no mesmo widget, como os problemas com o widget para informar data de nascimento na tela de inscrever-se (MAC-1, MAC-3, AH-14) na Figura 2, por exemplo; e

- os problemas estão relacionados a um mesmo objetivo do usuário ainda que tenham sido identificados em partes diferentes da interface e em métodos diferentes, como no caso dos problemas AH-18 (Figura 7) e MAC-9 (Figura 8) sobre o objetivo de consultar os detalhes de um evento, por exemplo.

Também foi possível identificar descuidos do designer ao revisar sua solução de IHC a partir dos problemas identificados na avaliação formativa. Alguns comportamentos indesejáveis do protótipo não foram corrigidos nos comentários do designer na melhoria da sua solução. Por exemplo, os problemas AH-11 e MAC-2 sobre valores padrão da data selecionada no widget de calendário (Figura 
3) não foram endereçados na descrição da solução correspondente. A falta de feedback sobre a busca de processos identificada no problema AH-17 também não foi endereçada nos comentários da respectiva solução no reprojeto. Muitos desses equívocos sobre o comportamento do sistema sendo projetado ocorreram em situações onde o designer teve que pensar em uma modificação da interface mais significativa. Diante de um esforço cognitivo maior no reprojeto da interface, o designer pode ter ignorado detalhes do comportamento do sistema por limitações cognitivas. Ele pode ter direcionado sua atenção para repensar a interface e acabou se esquecendo de repensar alguns aspectos da interação. É interessante observar que em situações em que o problema envolva apenas o comportamento do sistema e, portanto, exigia um esforço cognitivo menor do designer, ele não teve dificuldade em encaminhar soluções adequadas. Por exemplo, o designer encaminhou adequadamente a solução para os problemas AH-2 (Figura 1) e MAC12 (Figura 13) sobre o comportamento do sistema.

\section{CONSIDERAÇÕES FINAIS}

Este trabalho apresentou um estudo de caso sobre o uso de resultados de avaliação formativa durante o processo de design para a melhoria da solução digital sendo concebida. Os 52 problemas identificados pela avaliação heurística, método de avaliação de comunicabilidade de teste de usabilidade foram estratificados pelo local afetado na interface com usuário do protótipo avaliado. Os problemas identificados foram justapostos às respectivas modificações para melhoria da solução de IHC. Em geral, um problema encaminhou uma modificação na solução. Quando se tratavam dos mesmos problemas encontrados por métodos diferentes, ou ocorriam na mesma parte da interface (widget) ou eram relacionados ao mesmo objetivo, o designer considerou vários problemas em conjunto para repensar sua solução. Nestes casos, ele acabou deixando de considerar a necessidade de rever alguns comportamentos indesejáveis na sua solução original, pelo menos de forma explícita nos seus comentários sob a solução revisada. 
Apesar deste relato de estudo de caso ser um importante indício de como a avaliação formativa pode contribuir com o processo de design, o assunto está longe de ser esgotado. Trabalhos futuros deveriam realizar estudos semelhantes com outros designers, outros sistemas computacionais e outros contextos. A reflexão do designer durante a revisão da solução sendo concebida poderia ser analisada em mais detalhes. O que influencia as decisões do designer nesta etapa do processo? Como apoiar o designer adequadamente? Como evitar que coisas importantes passem despercebidas ou acabem sendo ignoradas diante da quantidade e complexidade de informações analisadas pelo designer? Futuras investigações sobre essas questões deveriam envolver: (1) uma melhor compreensão dos problemas encontrados em uma avaliação do uso de uma solução digital (sistema computacional interativo); (2) análise de impacto das condições em que a avaliação foi realizada, tais como cansaço e stress dos avaliadores, pressões sociais e demais fatores ambientais; (3) análise de custo/benefício de outros avaliadores revisarem os resultados da avaliação; (4) desenvolvimento de métodos e ferramentas (inclusive com suporte computacional de softwares e hardwares) para apoiar a análise do resultados de avaliação e a revisão da solução sendo concebida; e (5) analisar como as representações da solução original, dos resultados da avaliação e da solução revisada influenciam o processo de design na melhoria da solução sendo concebida. 
Projética, Londrina, v. 11, n. 2, p.80-103, agosto 2020

\section{REFERÊNCIAS}

1. BARBOSA, Simone Diniz Junqueira; SILVA, Bruno Santana da. Interação humano-computador. Rio de Janeiro, RJ: Elsevier Brasil, 2010. (Série Editora Campus. SBC- Sociedade Brasileira de Computação).

2. BIM, Sílvia Amélia; SALGADO, Luciana Cardoso de Castro; LEITÃO, Carla Faria. Evaluation by inspection: comparing methods of practical, cognitive and semiotic basis. In: BRAZILIAN SYMPOSIUM ON HUMAN FACTORS IN COMPUTING SYSTEMS, 15th, 2016, São Paulo, SP. Proceedings [...]. New York, NY: ACM, 2016. Article n. 9, p. 1-10. ISBN: 978-1-4503-5235-2.

3. JEFFRIES, Robin; MILLER, James R.; WHARTON, Cathleen; UYEDA, Kathy M. User interface evaluation in the real world: a comparison of four techniques. In: HUMAN FACTORS IN COMPUTING SYSTEMS CONFERENCE - CHI 91, New Orleans, 1991. Proceedings [...]. New York, NY: ACM, 1991. p. 119-124.

4. KARAT, Claire-Marie; CAMPBELL, Robert; FIEGEL, Tarra. Comparison of empirical testing and walkthrough methods in user interface evaluation. In: HUMAN FACTORS IN COMPUTING SYSTEMS CONFERENCE - CHI 92, 1992, Monterey, California. Proceedings [...]. New York, NY: ACM, 1992. p. 397-404.

5. LAWSON, Bryan R. How designers think: the design process demystified. 4th ed. Burlington, MA: Elsevier/Architectural, 2006.

6. NIELSEN, Jakob. Heuristic evaluation. In: MACK, Robert; NIELSEN, Jakob. (ed.). Usability inspection methods. New York, NY: John Wiley \& Sons, 1994. p. 25-62.
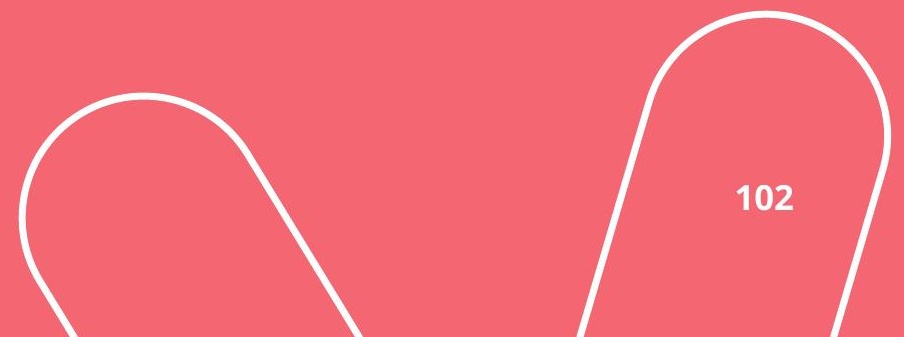
7. PRATES, Raquel O.; SOUZA, Clarisse Sieckenius de; BARBOSA, Simone Diniz Junqueira. A method for evaluating the communicability of user interfaces. ACM Interactions, New York, NY, v. 7, n. 1, p. 31-38, 2000.

8. RUBIN, Jeffrey; CHISNELL, Dana. Handbook of usability testing: how to plan, design, and conduct effective tests. 2. ed. Indianapolis, IN: Wiley Publishing, 2008.

9. SALGADO, Luciana Cardoso de Castro; BIM, Sílvia Amélia; SOUZA, Clarisse Sieckenius de. Comparação entre os métodos de avaliação de base cognitiva e semiótica. In: SIMPÓSIO SOBRE FATORES HUMANOS EM SISTEMAS COMPUTACIONAIS, 7., 2006, Natal, RN. Anais [...]. Natal, RN: IHC, 2006. p. 158-167.

10. SANTOS, Rafael Dias; SILVA, Bruno Santana da. Comparação de métodos de avaliação de IHC sob a perspectiva do autor da interface. In: CONGRESSO BRASILEIRO DE PESQUISA E DESENVOLVIMENTO EM DESIGN - P\&D, 13., 2018, Joinville, SC. Blucher Design Proceedings, São Paulo, SP, v. 6, n. 1, p. 5302-5316, mar. 2019. DOI: 10.5151/ped2018-7.2_ACO_06.

11. SCHÖN, Donald A. The reflective practitioner. New York: Basic Books, 1983.

12. SHARP, Helen; ROGERS, Yvonne; PREECE, Jennifer. Interaction design: beyond human-computer interaction. 2nd ed. New York, NY: John Wiley \& Sons, 2007.

13. STAKE, Robert E. The art of case study research. Thousand Oaks: Sage Publications, 1995.

14. YIN, Robert K. Applications of case study research. Thousand Oaks, CA: SAGE Publications, 2011.

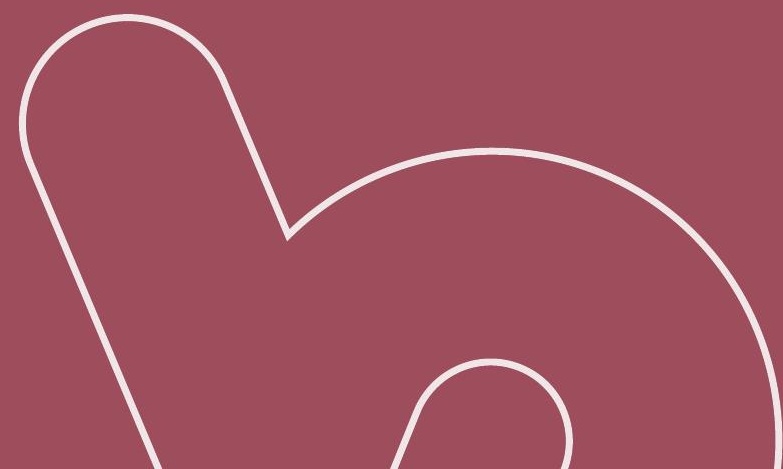

